\title{
Autophagy may protect MC3T3-E1 cells from fluoride-induced apoptosis
}

\author{
MIN WEI ${ }^{1}$, DONGMEI DUAN ${ }^{2}$, YUJIE LIU $^{1}$, ZHIGANG WANG $^{1}$ and ZHONGLI LI ${ }^{1}$ \\ Departments of ${ }^{1}$ Orthopaedics and ${ }^{2}$ Traditional Chinese Medicine, Chinese PLA General Hospital, Beijing 100853, P.R. China
}

Received June 23, 2013; Accepted February 4, 2014

DOI: $10.3892 / \mathrm{mmr} .2014 .2079$

\begin{abstract}
Fluoride is an essential trace element for all mammalian species; however, excess fluoride intake is known to be toxic to cells in animals and humans. The toxicity of fluoride is mainly exerted via induction of apoptosis. Autophagy is induced by numerous cytotoxic stimuli; however, it is often unclear whether, under specific conditions, autophagy has a pro-survival or a pro-apoptotic role. To answer this critical question, the present study assessed autophagy and apoptosis simultaneously in single cells. It was demonstrated that fluoride was able to inhibit cell proliferation and induce apoptosis and autophagy, whereas autophagy appeared to be protective. Further analysis revealed that MAPK/JNK-dependent autophagy may be protective in fluoride-induced apoptosis. It is anticipated that the presented single-cell approach may be a powerful tool for gaining a quantitative understanding of the complex regulation of autophagy, its effect on cell fate and its association with other cellular pathways.
\end{abstract}

\section{Introduction}

Fluoride is an essential trace element for all mammalian species; however, in excess, it is known to be toxic to animals and cultured cells (1). Excessive exposure to fluoride in drinking water, or in combination with exposure to fluoride from other sources, may give rise to a number of adverse effects, including dental fluorosis, skeletal fluorosis and vascular calcification (2-4). However, the cellular mechanisms underlying fluoride-induced cytotoxicity in osteoblasts remain to be fully elucidated. Apoptosis induced by fluoride has been demonstrated; however, to the best of our knowledge,

Correspondence to: Mr. Min Wei, Department of Orthopaedics, Chinese PLA General Hospital, 28 Fuxing Road, Beijing 100853, P.R. China

E-mail: weimin2012_2@163.com

Abbreviations: $\alpha$-MEM, $\alpha$-Minimal essential medium; MAPK, mitogen-activated protein kinase; 3-MA, 3-methyladenine; JNK, c-Jun N-terminal kinase; RAPA, autophagy-inducer rapamycin

Key words: apoptosis, autophagy, c-Jun N-terminal kinase, toxicity the occurrence of fluoride-induced autophagy has not been studied. The objectives of the present study were to determine the effects of fluoride treatment on the viability, apoptosis and autophagy of the osteoblastic cell line MC3T3-E1, the association between them.

Autophagy is an evolutionarily conserved lysosomal degradation process by which cells recycle damaged/obsolete macromolecules and organelles (5). Autophagy primarily fulfils a survival role during adaptation to unfavorable growth conditions or following cellular stress (6). Previous studies also demonstrated its involvement in general processes, including differentiation, development, defense against pathogens, ageing, apoptosis and cell death (7-10). Specifically, the association between autophagy and apoptosis is close and complex. Therefore, further studies to enhance the understanding of the molecular processes contributing to autophagy have provided insight into the association between autophagy and apoptosis. Recently studies suggest that autophagy serves a largely cytoprotective role under physiologically relevant conditions $(11,12)$. The cytoprotective function of autophagy is mediated by the negative modulation of apoptosis (13); however, the mechanisms mediating the complex counter-regulation of apoptosis and autophagy are have yet to be fully elucidated.

In the present study, it was demonstrated that fluoride induces apoptosis as well as autophagy in MC3T3-E1 cells. Fluoride-induced autophagy may protect MC3T3-E1 cells against fluoride-induced apoptosis. It was also demonstrated that the expression of phosphorylated c-Jun N-terminal kinase (p-JNK) was upregulated by fluoride, which contributes to the induction of autophagy. These results offer an inference that drugs targeting autophagy may improve therapy for fluoride damage.

\section{Materials and methods}

Reagents. $\alpha$-Minimal essential medium ( $\alpha$-MEM), penicillin-streptomycin $(5,000 \mathrm{U} / \mathrm{ml}$ penicillin; $5,000 \mathrm{U} / \mathrm{ml}$ streptomycin), fetal bovine serum (FBS), rapamycin (RAPA) and 3-methyladenine (3-MA) were obtained from Invitrogen (Carlsbad, CA, USA). MTT dimethylsulfoxide (DMSO) and Triton X-100 were purchased from Sigma (St. Louis, MO, USA). Fluoride was obtained from Xinhua Pure Chemical Industries (Shenyang, China). All reagents used were trace element analysis grade. All water used was glass distilled. 
Cell culture. Osteoblastic MC3T3-E1 cells (CRL-2593; American Type Culture Collection, Manassas, VA, USA) were cultured in regular growth medium ( $\alpha$-MEM with $10 \% \mathrm{FBS}$, and $1 \%$ penicillin/streptomycin) at $37^{\circ} \mathrm{C}$ in a $5 \% \mathrm{CO}_{2}$ incubator. They were subcultured every three days using $0.2 \%$ trypsin plus $0.02 \%$ EDTA. For experiments, cells were cultured for $24 \mathrm{~h}$ to obtain monolayers containing $3 \mathrm{ml} \alpha$-MEM with $10 \%$ FBS. Following rinsing of the cells with phosphate-buffered saline (PBS), the medium was exchanged with medium containing either sodium fluoride $(\mathrm{NaF})$ or other agents and the cells were further cultured.

MTT assay. Cell proliferation by treatment with various concentrations of NaF during 24-72 h was detected by MTT assay. Briefly, MC3T3-E1 cells were seeded into 96-well plates $\left(6 \times 10^{3}\right.$ cells/well) and maintained in growth media for $24 \mathrm{~h}$ under $5 \% \mathrm{CO}_{2}$ at $37^{\circ} \mathrm{C}$. At $60 \%$ confluence, the cells were treated with $(0-10 \mathrm{mmol} / \mathrm{l}) \mathrm{NaF}$ for 24,48 and $72 \mathrm{~h}$, respectively. Thereafter, $10 \mu \mathrm{l}$ of MTT solution $(5 \mathrm{mg} / \mathrm{ml})$ was added to each well and the cells were incubated for another $4 \mathrm{~h}$ at $37^{\circ} \mathrm{C}$. Following the formation of formazan crystals, MTT medium was then replaced with $150 \mu \mathrm{l}$ of DMSO for dissolving the formazan crystals and the plates were agitated for $5 \mathrm{~min}$. The absorbance of each well was recorded with a microplate spectrophotometer at $570 \mathrm{~nm}$. Relative cellular growth was determined from the ratio of the average absorbance in treated cells versus the average absorbance in control cells. The cell viability was calculated as the ratio of optical densities.

Analysis of apoptosis. MC3T3-E1 cells were cultured at $4 \times 10^{6}$ cells $/ \mathrm{ml}$ and seeded in six-well plates. Cells were harvested by trypsinization, then washed twice with cold PBS and centrifuged at $110 \times \mathrm{g}$. Cells $\left(1 \times 10^{5}-1 \times 10^{6}\right)$ were then resuspended in $300 \mu \mathrm{l}$ of $1 \mathrm{X}$ binding buffer, centrifuged again at $110 \mathrm{x} \mathrm{g}$ for $5 \mathrm{~min}$ and then the supernatant was removed. Cells were resuspended in $300 \mu \mathrm{l}$ of $1 \mathrm{X}$ binding buffer and transferred to a sterile flow cytometry glass tube. $10 \mu \mathrm{l}$ of Annexin V-fluorescein isothiocyanate (FITC) was added and cells were incubated in the dark for $30 \mathrm{~min}$ at room temperature. Then cells were incubated in the dark with $5 \mu \mathrm{l}$ propidium iodide (PI) and analyzed using a flow cytometer (FACSCalibur, Becton-Dickinson, Franklin Lakes, NJ, USA). Cellular apoptosis was determined using the Annexin V-FITC Apoptosis Detection kit I (Clontech Laboratories Inc, Mountain View, CA, USA).

Terminal deoxynucleotidyl transferase dUTP nick end labeling (TUNEL) method. Following incubation with $1 \%$ paraformaldehyde for $5 \mathrm{~min}$, cells were fixed in 2:1 v/v ethanol - acetic acid for $10 \mathrm{~min}$ at room temperature. Following three washes with PBS, cells were incubated with $100 \mathrm{U} / \mathrm{ml}$ terminal deoxyuridine transferase, $0.5 \mu \mathrm{g} / \mathrm{ml}$ biotinylated uridine in $1 \mathrm{M}$ potassium cacodylate and $125 \mathrm{mM}$ Tris- $\mathrm{HC} 1,2.5 \mathrm{mM}$ cobalt chloride (Boeringher, Mannheim, Germany), at pH 6.6 for $1 \mathrm{~h}$ at $37^{\circ} \mathrm{C}$ in a humidified chamber. Following washes, a 1:40 solution of fluoresceinated streptavidin (Boeringher) was incubated for $30 \mathrm{~min}$ at room temperature. Slides were counter-stained with $0.3 \mu \mathrm{g} / \mathrm{ml}$ of PI (Sigma) in PBS for $1 \mathrm{~min}$ at room temperature. An epifluorescent microscope (Ernst Leitz, Inc., Rockleigh, NJ, USA) was used to detect apoptotic cells, which were quantified by counting the number of fluorescein-positive cells relative to the total number of cells in at least 10 microscopic fields. For each experiment 200 cells were counted.

Preparation of proteins in the mitochondrial and cytosolic fractions and western blot analysis. The methods for preparation of proteins in the mitochondrial and cytosolic fractions and western blot analysis have been described previously (14). The cells were washed twice in ice-cold PBS and resuspended in five volumes of ice-cold extraction buffer $(20 \mathrm{mM}$ western blotting Hepes-KOH, $1.5 \mathrm{mM} \mathrm{MgCl}_{2}, 1 \mathrm{mM}$ EDTA, $1 \mathrm{mM}$ ethylene glycol tetraacetic acid, $1 \mathrm{mM}$ dithiothreitol and $0.1 \mathrm{mM}$ phenylmethanesulfonyl fluoride, $\mathrm{pH}$ 7.5. The resuspended cells were homogenized with ten strokes of a Teflon homogenizer. The homogenates were centrifuged twice at $750 \mathrm{x} \mathrm{g}$ for $10 \mathrm{~min}$ at $4^{\circ} \mathrm{C}$. The supernatants were centrifuged at $10,000 \mathrm{x}$ g for $15 \mathrm{~min}$ at $4^{\circ} \mathrm{C}$ to obtain the mitochondrial pellets. Cytosolic fractions were obtained following further centrifugation at $100,000 \mathrm{x}$ for $1 \mathrm{~h}$ at $4^{\circ} \mathrm{C}$. The protein concentrations of the resulting supernatants and mitochondrial fractions were measured. The samples (10 $\mu \mathrm{g}$ protein) were separated by sodium dodecyl sulfate polyacrylamide gel electrophoresis with 10 and $8 \%$ polyacrylamide gel. The following primary antibodies were used: anti-B-cell lymphoma 2-associated x (Bax), anti-apoptosis-inducing factor (AIF), anti-cytochrome-C, anti- $\beta$-actin (Santa Cruz Biotechnology, Inc., Santa Cruz, CA, USA), anti-caspase 3, anti-microtubule-associated protein 1 light chain $3 \alpha$ (LC3) and anti-Beclin 1 (Cell Signaling Technology, Inc., Danvers, MA, USA). These separated proteins in SDS-PAGE were electrotransferred onto a Hybond-polyvinylidene fluoride (PVDF) membrane. The individual SDS gels were distinguished by placing the protein molecular weight markers (Invitrogen Life Technologies, Carlsbad, CA, USA) in a different but consistent position. The PVDF membrane was then soaked in a blocking solution (5\% nonfat milk in TBST buffer; $20 \mathrm{mM}$ Tris-HCl, $\mathrm{pH} 7.5,0.5 \mathrm{M} \mathrm{NaCl}, 0.1 \%$ Tween 20) for $2 \mathrm{~h}$ at room temperature. The soaked PVDF membrane was then incubated in Tris-buffered saline/Tween 20 (TBST) containing primary antibodies overnight at $4^{\circ} \mathrm{C}$, washed with TBST buffer three times for $5 \mathrm{~min}$ each and incubated at room temperature for $2 \mathrm{~h}$ in TBST containing horseradish peroxidase (HRP)-conjugated goat anti-mouse and goat anti-rabbit immunoglobulin $\mathrm{G}$ (IgG) (Santa Cruz Biotechnology, Inc.). The membrane was washed with TBST buffer three times for 10 min each. The membranes were incubated with enhanced chemoluminescence reagent (Pierce Biotechnology, Inc., Rockford, IL, USA) for HRP (30 sec) and exposed to autoradiography films for visualization of the bands. The relative amounts of various proteins were analyzed. The results were quantified by Quantity One Software.

Fluorescence microscopy. MC3T3-E1 cells were seeded into six-well plates and incubated for $24 \mathrm{~h}$. Then cells were washed once with ice-cold PBS and fixed with $4 \%$ paraformaldehyde for $30 \mathrm{~min}$ at $4^{\circ} \mathrm{C}$. Following washing with PBS three times, the cells were incubated with $1 \%$ Triton X-100 for $10 \mathrm{~min}$. The cells were blocked at nonspecific antibody binding sites by incubating with $10 \%$ goat serum in PBS containing 
$0.3 \%$ Triton $\mathrm{X}-100$ and $0.5 \%$ bovine serum albumin for $30 \mathrm{~min}$ at room temperature, followed by incubation with a mouse monoclonal antibody against Beclin 1 (1:400 in PBS, Cell Signaling Technology) overnight. Cells were incubated with FITC-conjugated goat anti-mouse $\operatorname{IgG}(1: 100$ in PBS) for $0.5 \mathrm{~h}$ at room temperature. Heochst 33342 was added to the cells for 15 min. Following washing three times with PBS, cells were visualized using fluorescence microscopy.

Statistical analysis. Statistical analysis was performed using SPSS (Version 17.0; SPSS, Inc., Chicago, IL, USA). The experiments were repeated at least three times. Data are expressed as the mean \pm standard deviation. Differences in the results for the two groups were evaluated by the Student's t-test. $\mathrm{P}<0.05$ was considered to indicate a statistically significant difference.

\section{Results}

Fluoride induces MC3T3-E1 cell apoptosis via a mitochondrial-mediated pathway. The effects of fluoride on osteoblastic MC3T3-E1 cells and the mechanism(s) by which fluoride affected MC3T3-E1 cell apoptosis were assessed at different concentrations. Osteoblastic MC3T3-E1 cells were treated with fluoride at concentrations ranging from 0 to $10 \mathrm{mM}$ for 24,48 or $72 \mathrm{~h}$, respectively. Cell viability assays demonstrated that fluoride inhibited cell growth in a dose- and time-dependent manner (Fig. $1 \mathrm{~A})$. The $\mathrm{IC}_{50}$-values of fluoride at 24, 48 and $72 \mathrm{~h}$ were 3.51, 5.02 and $6.36 \mathrm{mM}$. Cells were treated with $5.0 \mathrm{mM}$ fluoride for 0,12 and $24 \mathrm{~h}$ and apoptosis was determined by flow cytometry followed by Annexin V/PI double staining. Flow cytometry assays demonstrated a substantial increase in the apoptotic population among cells treated with fluoride at 12 and $24 \mathrm{~h}$ (Fig. 1B). From the results of the TUNEL assay, it was demonstrated that apoptosis was induced following incubation of MC3T3-E1 cells with fluoride. To further confirm that apoptosis was induced by fluoride, western blot analysis was performed to detect the expression of AIF (in cytosol), cytochrome-C (in cytosol) and Bax (in the mitochondria) as well as caspase 3. As displayed in Fig. 1D, the expression of Bax in mitochondria was significantly increased in the fluoride groups compared with the control group $(\mathrm{P}<0.01)$, suggesting that the translocation of Bax into the mitochondria was involved in cell death induced by fluoride. Mitochondria-mediated apoptosis comprises caspase-dependent and caspase-independent processes, while AIF is involved in the caspase-independent response. As displayed in Fig. 1D, the expression of AIF and cytochrome-C in the cytosol were significantly increased in the fluoride groups compared with the control group $(\mathrm{P}<0.01)$ while the expression of Bax (in the mitochondria) was upregulated in the fluoride group. These results demonstrate that the release of AIF from the mitochondria into the cytosol was involved in cell death. Similarly, caspase-3 was examined by western blot analysis. Levels of cleaved caspase 3 in fluoride groups were upregulated compared with the control group (Fig. 1F). These data indicated that fluoride-induced apoptosis in MC3T3-E1 cells proceeded via a mitochondrial pathway involving the caspase-dependent (caspase 3 ) and the caspase-independent (AIF) pathways.
Fluoride induces MC3T3-E1 cell autophagy. To determine whether mitochondrial damage was a cause or consequence of autophagy, cells were treated with $5.0 \mathrm{mM}$ of fluoride for $0,4,8,12,16$ and $24 \mathrm{~h}$ and the expression of Beclin 1 and Lc3-2/1 were detected by western blot analysis. The occurrence of autophagy following exposure of cells to fluoride was assessed. As shown in Fig. 2A and B, the levels of Lc3-2/1 began to increase after $8 \mathrm{~h}$, peaked at $12 \mathrm{~h}$, remained at this level until $16 \mathrm{~h}$ and decreased after $16 \mathrm{~h}$ of incubation. Similar changes were observed in regard to the expression of Beclin 1, suggesting that fluoride not only induced apoptosis but also induced autophagy in MC3T3-E1 cells. Cultured with fluoride, autophagy was quickly activated along with apoptosis. From the results of fluorescence microscopy, fluoride led to enhanced levels of Beclin 1, with induction being sustained upon fluoride stimulation compared with the control (Fig. 2C). The fluorescence intensity in the $12 \mathrm{~h}$ group was greater than that in the $24 \mathrm{~h}$ group. These findings indicated that fluoride did not block autophagic flux, but induced autophagic activity.

Fluoride treatment activates JNK to induce autophagy. Studies have shown that JNK activation promotes autophagy in different cell lines $(15,16)$. To investigate the role of JNK in fluoride-induced autophagy, MC3T3-E1 cells were treated with $5.0 \mathrm{mM}$ fluoride for $0-24 \mathrm{~h}$ and the JNK level was detected using western blotting. As displayed in Fig. 3A, incubation with fluoride increased the phosphorylation of JNK from 8 to $16 \mathrm{~h}$. However, the co-treatment with $1 \mu \mathrm{M}$ SP600125 (an inhibitor of the MAPK/JNK pathway; concentration from reference 17), effectively inhibited JNK phosphorylation and suppressed fluoride-induced LC3-2 protein (Fig. 3C). These results suggest that the MAPK/JNK pathway is involved in to the autophagy of MC3T3-E1 cells induced by fluoride. Fluoride-induced autophagy is mediated by JNK activation.

Autophagy induced by fluoride protects MC3T3-E1 cells from undergoing apoptosis. To investigate the effect of autophagy on apoptosis, 3-methyladenine (3-MA), a potent pharmacological inhibitor of autophagy, was used to suppress fluoride-induced autophagy. It was demonstrated that pretreatment with $5 \mathrm{mM}$ 3-MA (the concentration of 3-MA from reference 18 was used) was able to block autophagy in MC3T3-E1 cells without significant cytotoxicity. 3-MA itself scarcely induced apoptosis and cell death; however it significantly increased apoptosis at $12 \mathrm{~h}$ following fluoride exposure (fluoride group: 17.5\%; fluoride plus 3-MA: 23.8\%) (Fig. 4A). These results suggested that suppression of autophagy by 3-MA was able to increase fluoride-induced injury in MC3T3-E1 cells. By contrast, fluoride-induced apoptosis decreased following co-treatment with $2 \mu \mathrm{M}$ of the autophagy-inducer RAPA (the concentration of RAPA from reference 19 was used) relative to cells treated with fluoride alone (Fig. 4A) (fluoride group: 17.5\%; fluoride plus RAPA: $13.2 \%$ ). Treatment of normal MC3T3-E1 cells with RAPA or 3-MA alone did not affect cell viability (Fig. 4A). The western blot analysis offered further evidence. The inhibitory effect of 3-MA the inductive effect of RAPA on autophagy in fluoride-induced apoptosis were also evaluated by western blot analysis. The upregulation of the expression of the apoptotic protein AIF (in the cytosol), cytochrome-C (in the 
A

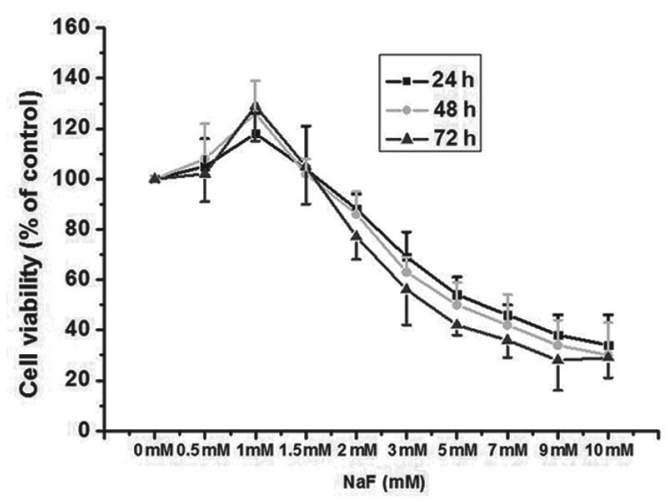

B

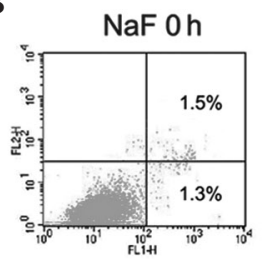

C

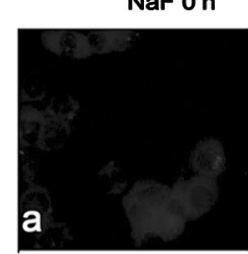

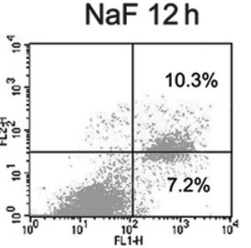

$\mathrm{NaF} 12 \mathrm{~h}$

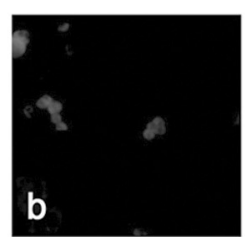

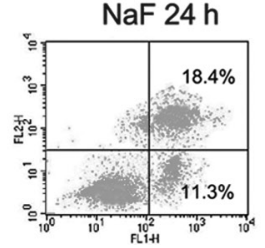

$\mathrm{NaF} 24 \mathrm{~h}$

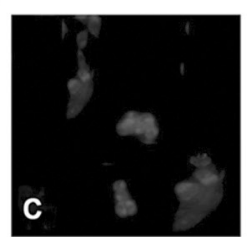

D

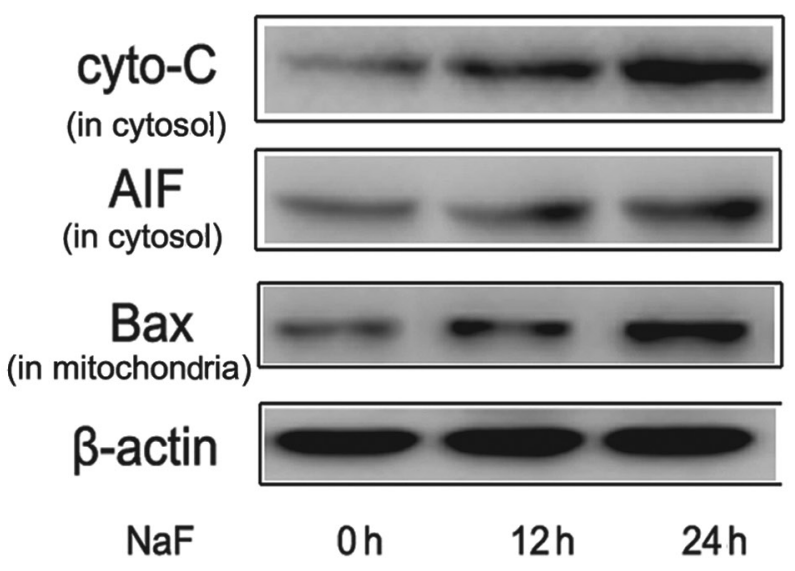

$\mathbf{E}$

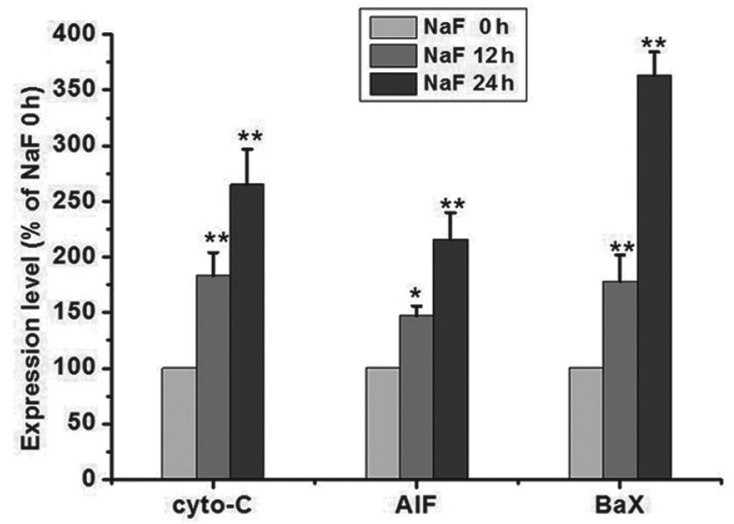

G

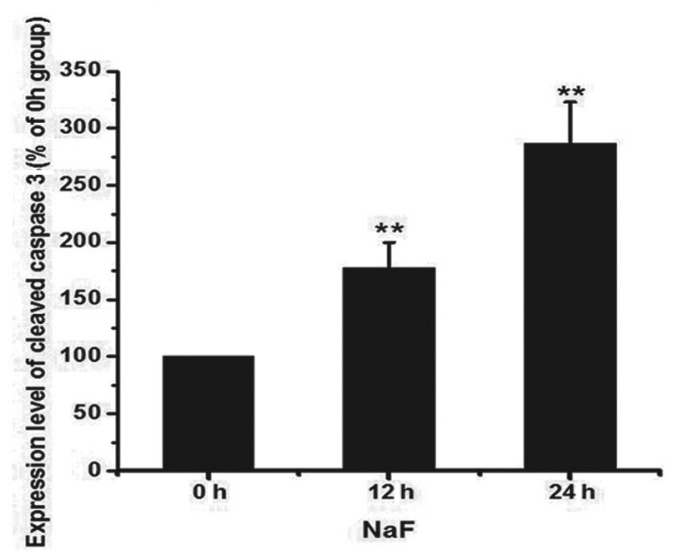

Figure 1. Effects of fluoride on the apoptosis of MC3T3-E1 cells. (A) MC3T3-E1 cells were treated with various doses of fluoride (0-10 mM) for 24, 48 or $72 \mathrm{~h}$ and the cell viability was analyzed by an MTT assay. Values represent the mean \pm standard deviation of three independent experiments. (B) Cells were treated with $5.0 \mathrm{mM}$ fluoride for 0,12 and $24 \mathrm{~h}$ and apoptosis was determined by flow cytometry followed by Annexin V-PI double staining. (C) Cells were treated as described and apoptosis was assessed by using terminal deoxynucleotidyl transferase dUTP nick end labeling analysis. Results are a representative of three experiments. (D) Cells were treated as described and the expression of cyto-C (in the cytosol), Bax (in the mitochondria) and AIF (in the cytosol) was detected by western blotting. (E) The results are representative of three independent experiments. $\beta$-actin was used as a loading control (** $\mathrm{P}<0.01 \mathrm{vs}$. control). (F) Cells were treated as described and the expression of caspase 3 was detected by western blotting. (G) The results were representative of three independent experiments. $\beta$-actin was used as a loading control ( ${ }^{* * *} \mathrm{P}<0.01$ vs. control). AIF, apoptosis inducing factor; NaF, sodium fluoride; cyto-C, cytochrome C; PI, propidium iodide; Bax, B-cell lymphoma 2 associated $\mathrm{X}$.

cytosol), Bax (in the mitochondria) and cleaved caspase 3 were more apparent when cells were co-treated with 3-MA but were attenuated by co-treatment with RAPA, as shown in Fig. 4B-E. These results indicate that autophagy induced by fluoride served a protective function and blockage of autophagy enhanced the apoptotic effect of fluoride in MC3T3-E1 cells.

\section{Discussion}

Fluoride is an essential trace element, which has been demonstrated to increase bone formation and promote cell proliferation in low concentrations for all mammalian species (20). Previous studies demonstrated that fluoride leads to the proliferation of osteoblasts, promotes their differentiation and modulates 
A

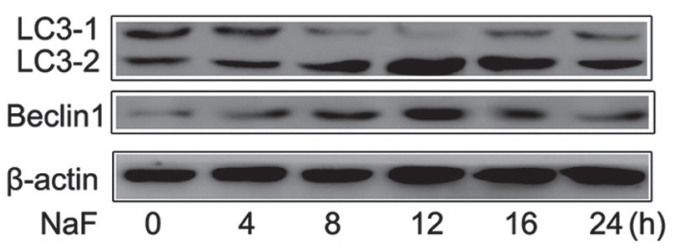

B

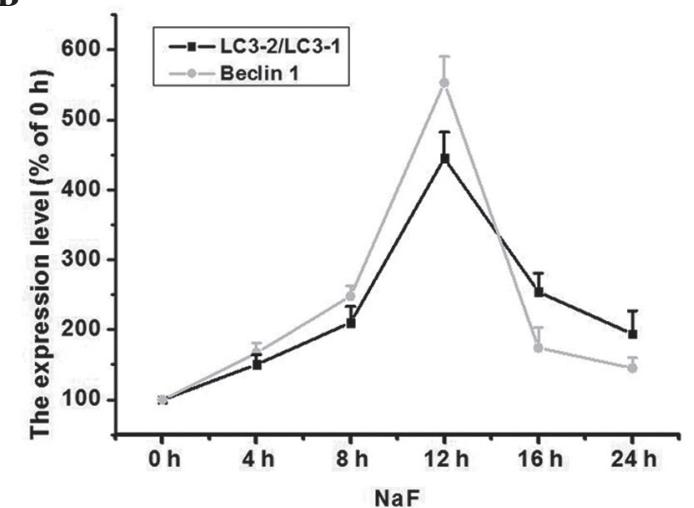

C

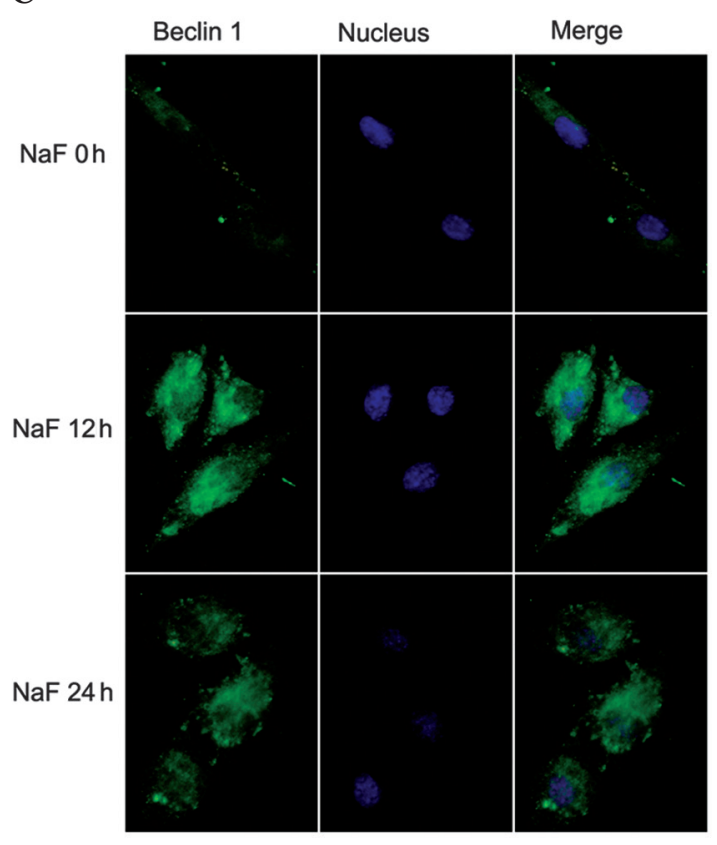

Figure 2. Effects of fluoride on the autophagy of MC3T3-E1 cells. (A-B) Cells were treated with $5.0 \mathrm{mM}$ fluoride for 0-24 h and the expression of Beclin 1 and Lc3-2/1 were detected by western blot analysis. The results were representative of three independent experiments. $\beta$-actin was used as a loading control. (C) Cells were treated with $5.0 \mathrm{mM}$ fluoride for 0,12 and $24 \mathrm{~h}$ and the Beclin 1 protein was stained and observed under a fluorescence microscope. NaF, sodium fluoride; LC3, microtubule-associated protein 1 light chain $3 \alpha$.

A

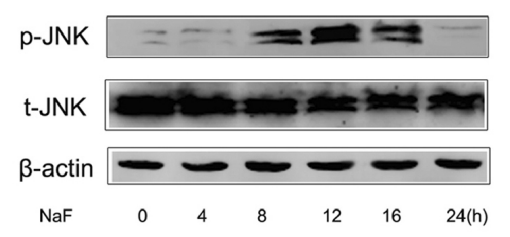

C

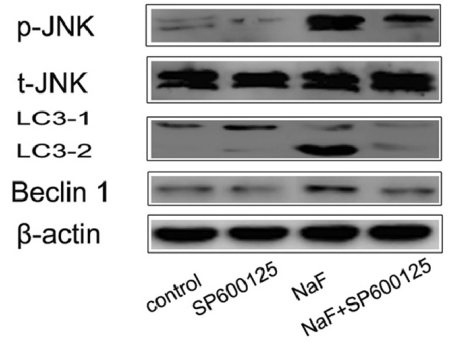

B

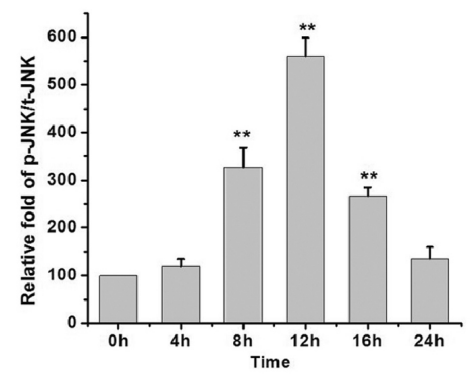

D

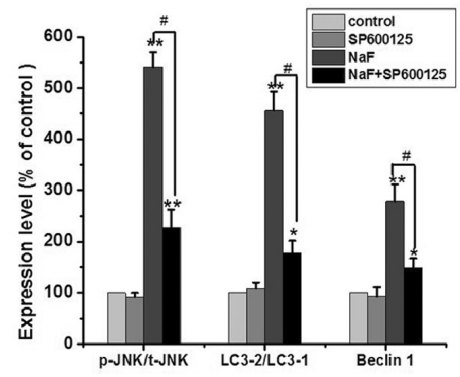

Figure 3. Participation of JNK in fluoride-induced autophagy. (A) MC3T3-E1 cells were treated with $5.0 \mathrm{mM}$ fluoride for the indicated time periods and then analyzed using wesern blot analysis with anti-p-JNK and anti-t-JNK antibodies. Image is a representative of three independent experiments. $\beta$-actin was used as a loading control. (B) Quantification of A $\left({ }^{* * *} \mathrm{P}<0.01\right.$ vs.control). (C) MC3T3-E1 cells were pretreated with $10 \mathrm{mM}$ of SP600125 for $1 \mathrm{~h}$, followed by treatment with or without fluoride for $12 \mathrm{~h}$ (for analysis of JNK and LC3). Cell lysates were analyzed by immunoblotting with anti-p-JNK, anti-t-JNK, Beclin 1 and anti-LC3. The results were representative of three independent experiments. $\beta$-actin was used as a loading control (D) Quantification of C $\left({ }^{* *} \mathrm{P}<0.01\right.$ vs. control; ${ }^{*} \mathrm{P}<0.05$ vs. control; \# $\mathrm{P}<0.05$ vs. NaF group). $\mathrm{p}$-JNK, phosphorylated c-Jun N-terminal kinase; LC 3 , microtubule-associated protein 1 light chain $3 \alpha$. 
A

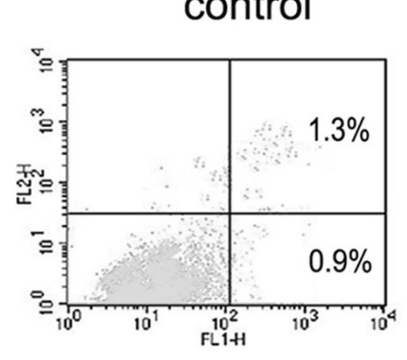

$\mathrm{NaF}$

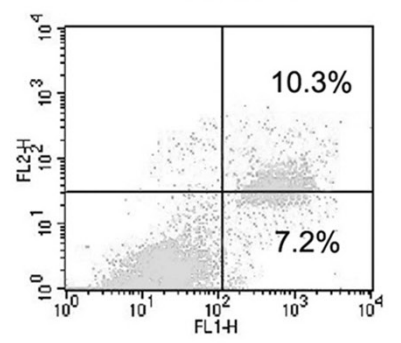

RAPA

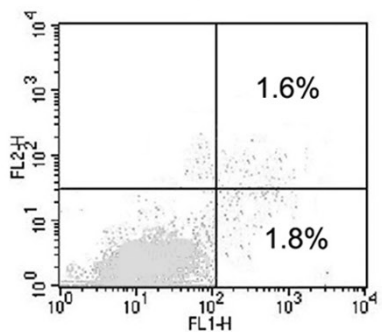

$\mathrm{NaF}+\mathrm{RAPA}$

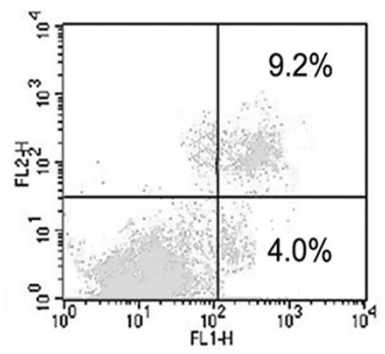

3-MA
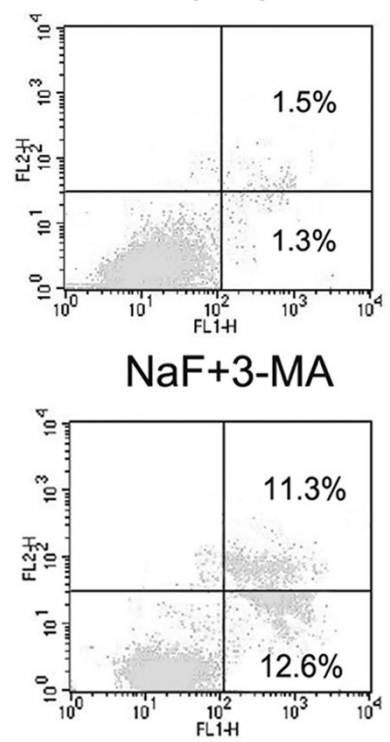

B

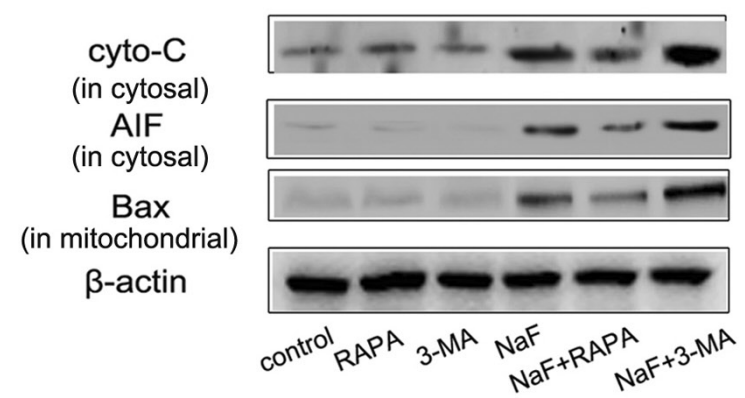

D

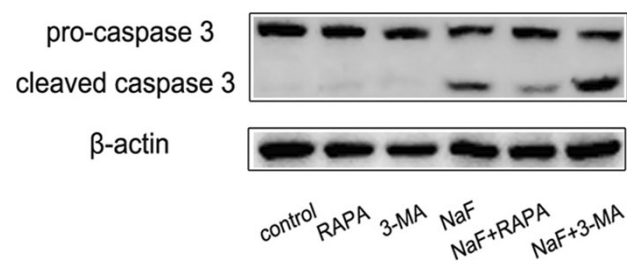

$\mathbf{C}$

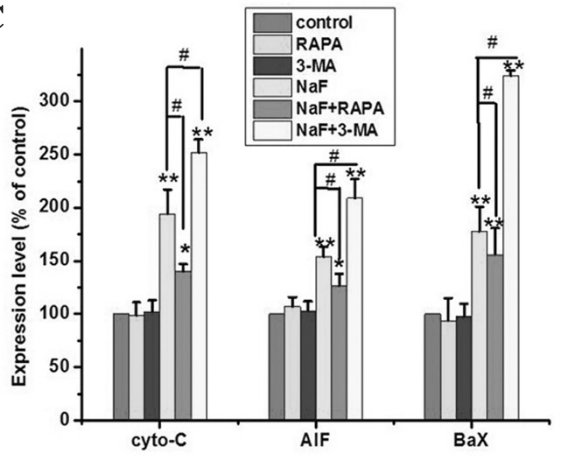

$\mathbf{E}$

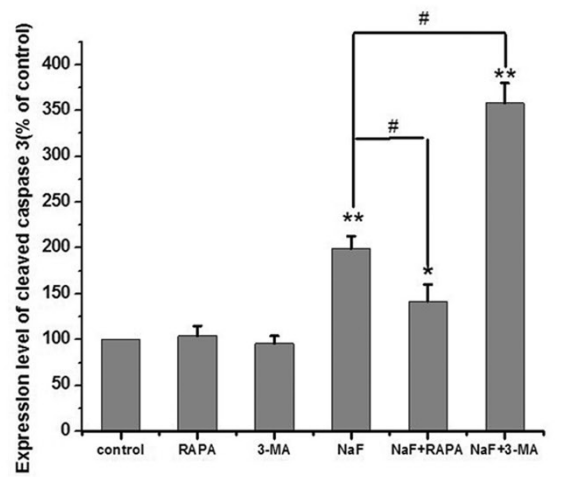

Figure 4. Autophagy had a protective function in MC3T3-E1 cells. (A) Cells were treated with $5.0 \mathrm{mM}$ of fluoride or $5 \mathrm{mM}$ of 3-MA, as well as $2 \mu \mathrm{M}$ of RAPA for $12 \mathrm{~h}$ or a combined treatment of $5.0 \mathrm{mM}$ fluoride and $5 \mathrm{mM}$ of 3-MA or $5.0 \mathrm{mM}$ fluoride and $2 \mu \mathrm{M}$ RAPA for $12 \mathrm{~h}$. Apoptosis was determined by flow cytometry followed by Annexin V-propidium iodide double staining. (B) Cells were treated as described and the expression of cyto-C (in the cytosol), Bax (in the mitochondria) and AIF (in the cytosol) was detected by western blot analysis. (C) The results were representative of three independent experiments. $\beta$-actin was used as a loading control. ( ${ }^{* *} \mathrm{P}<0.01$ vs. control; ${ }^{*} \mathrm{P}<0.05$ vs. control; \#P $<0.05$ vs. NaF group). (D) Cells were treated as described and the expression of caspase 3 was detected by western blot analysis. (E) The results were representative of three independent experiments. $\beta$-actin was used as a loading control. $\left({ }^{* *} \mathrm{P}<0.01\right.$ vs. control; " $\mathrm{P}<0.05$ vs. control; " $\mathrm{P}<0.05$ vs. NaF group). 3-MA, 3-methyladenine; RAPA, rapamycin; AIF, apoptosis inducing-factor; NaF, sodium fluoride; cyto-C, cytochrome C; Bax, B-cel lymphoma 2-associated X.

the activity of growth factors, including insulin-like growth factor (20). Fluoride also increases alkaline phosphatase-specific activity and promotes bone formation in rats in vivo (21). However, excess fluoride may give rise to a number of adverse effects. Studies have demonstrated that excess fluoride is able to downregulate the expression of the collagen I gene and induce apoptosis in newborn rat osteoblasts (22). The expression of insulin-like growth factor-I was also decreased when cultured with high concentrations of fluoride in mouse osteoblasts (23). Fluoride was able to induce osteoblast cell apoptosis; however, the mechanism of apoptosis was not clear. The expression of Bax is closely associated with mitochondrial apoptosis, which 
is important in the regulation of cell apoptosis (24). The present study demonstrated that fluoride increased the expression of Bax. Thus, this type of apoptosis is a mitochondria-mediated apoptosis. Mitochondria-mediated apoptosis comprises caspase-dependent and caspase-independent processes. Cytochrome- $\mathrm{C}$ is linked to caspase-dependent apoptotic signaling, whereas AIF is involved in the caspase-independent response (25). The present study demonstrated that the levels of the two proteins significantly increased in MC3T3-E1 cells induced by fluoride. Therefore, it is hypothesized that apoptosis caused by fluoride appears to proceed via mitochondrial dysfunction linked to caspase-dependent/-independent pathways.

The link between apoptosis and autophagy is complex and difficult to elucidate. The process of apoptosis is often accompanied by the occurrence of autophagy (26). The present study demonstrated that fluoride not only induced apoptosis but also induced autophagy in MC3T3-E1 cells. As displayed in Fig. 2, the autophagy proteins, including Beclin 1 and LC3, were upregulated by fluoride. Furthermore, in accordance with previous studies, the present study revealed that fluoride activated autophagy by initiation of the JNK signaling pathway (27). Autophagy is generally considered to be a survival mechanism of cells (28). It was demonstrated that the suppression of autophagy by 3-MA was able to increase fluoride-induced injury in MC3T3-E1 cells. By contrast, fluoride-induced apoptosis was decreased following co-treatment with the autophagy-inducer RAPA. The results suggest that autophagy is a pro-survival mechanism for fluoride-treated MC3T3-E1 cells and reduces the incidence of apoptosis, facilitating the survival of the cells. Several mechanisms for autophagy-limiting apoptosis have been discussed. For example, the release of B-cell lymphoma 2 and FLICE-like inhibitory protein (FLIP: A member of the tumour necrosis factor signaling pathway and a regulator of apoptosis) from autophagocytosis-associated protein Atg3 protein complexes may inhibit the pathways of apoptosis (29). Hypoxia-inducible factor and autophagy are closely correlated and may be important in anti-apoptotic signaling (30). However, the precise mechanism for autophagy-mediated anti-apoptosis remains to be elucidated and is under investigation in our laboratory.

\section{References}

1. Alonso Á and Camargo JA: A video-based tracking analysis to assess the chronic toxic effects of fluoride ion on the aquatic snail Potamopyrgus antipodarum (Hydrobiidae, Mollusca). Ecotoxicol Environ Saf 81: 70-75, 2012.

2. Mascarenhas AK: Risk factors for dental fluorosis: a review of the recent literature. Pediatr Dent 22: 269-277, 2000.

3. Rawlani S, Rawlani S and Rawlani S: Assessment of Skeletal and Non-skeletal Fluorosis in Endemic Fluoridated Areas of Vidharbha Region, India: A Survey. Indian J Community Med 35: 298-301, 2010.

4. Li Y, Berenji GR, Shaba WF, Tafti B, et al: Association of vascular fluoride uptake with vascular calcification and coronary artery disease. Nucl Med Commun 33: 14-20, 2012.

5. Stroikin Y, Dalen H, Lööf S, et al: Inhibition of autophagy with 3 -methyladenine results in impaired turnover of lysosomes and accumulation of lipofuscin-like material. Eur J Cell Biol 83: 583-590, 2004

6. Dutta D, Xu J, Kim JS, et al: Upregulated autophagy protects cardiomyocytes from oxidative stress-induced toxicity. Autophagy 9: 328-344, 2013.
7. Jacquel A, Obba S, Boyer L, et al: Autophagy is required for CSF-1-induced macrophagic differentiation and acquisition of phagocytic functions. Blood 119: 4527-4531, 2012.

8. Graziotto JJ, Cao K, Collins FS, et al: Rapamycin activates autophagy in Hutchinson-Gilford progeria syndrome: implications for normal aging and age-dependent neurodegenerative disorders. Autophagy 8: 147-151, 2012.

9. Joubert PE, Werneke SW, de la Calle C, et al: Chikungunya virus-induced autophagy delays caspase-dependent cell death. J Exp Med 209: 1029-1047, 2012.

10. Zhou F, Yang Y and Xing D: Bcl-2 and Bcl-xL play important roles in the crosstalk between autophagy and apoptosis. FEBS J 278: 403-413, 2011.

11. Bhogal RH, Weston CJ, Curbishley SM, et al: Autophagy: a cyto-protective mechanism which prevents primary human hepatocyte apoptosis during oxidative stress. Autophagy 8: 545-558, 2012.

12. Hui B, Shi YH, Ding ZB, et al: Proteasome inhibitor interacts synergistically with autophagy inhibitor to suppress proliferation and induce apoptosis in hepatocellular carcinoma. Cancer 118: 5560-5571, 2012

13. Ponnusamy M, Liu N, Sellamuthu R, et al: Autophagy protects against necrotic renal epithelial cell-induced death of renal interstitial fibroblasts. Am J Physiol Renal Physiol 303: F83-F91, 2012.

14. Wessel D and Flügge UI: A method for the quantitative recovery of protein in dilute solution in the presence of detergents and lipids. Anal Biochem 138: 141-143, 1984.

15. Xu P, Das M, Reilly J, et al: JNK regulates FoxO-dependent autophagy in neurons. Genes Dev 25: 310-322, 2011.

16. Komiya K, Uchida T, Ueno T, et al: Free fatty acids stimulate autophagy in pancreatic $\beta$-cells via JNK pathway. Biochem Biophys Res Commun 401: 561-567, 2010.

17. Yang CM, Chien CS, Yao CC, et al: Mechanical strain induces collagenase-3 (MMP-13) expression in MC3T3-E1 osteoblastic cells. J Biol Chem 279: 22158-22165, 2004.

18. Zeng Y, Yang X, Wang J, et al: Aristolochic acid I induced autophagy extenuates cell apoptosis via ERK 1/2 pathway in renal tubular epithelial cells. PLoS One 7: e30312, 2012.

19. Amarnath S, Flomerfelt FA, Costanzo CM, et al: Rapamycin generates anti-apoptotic human Th1/Tc1 cells via autophagy for induction of xenogeneic GVHD. Autophagy 6: 523-541, 2010.

20. Ren G, Wang K, Chang R, et al: Simultaneous administration of fluoride and selenite regulates proliferation and apoptosis in murine osteoblast-like MC3T3-E1 cells by altering osteoprotegerin. Biol Trace Elem Res 144: 1437-1448, 2011.

21. Mousny M, Omelon S, Wise L, et al: Fluoride effects on bone formation and mineralization are influenced by genetics. Bone 43: 1067-1074, 2008.

22. Yan X, Yan X, Morrison A, et al: Fluoride induces apoptosis and alters collagen I expression in rat osteoblasts. Toxicol Lett 200: 133-138, 2011.

23. Wang Z, Yang X, Yang S, et al: Sodium fluoride suppress proliferation and induce apoptosis through decreased insulin-like growth factor-I expression and oxidative stress in primary cultured mouse osteoblasts. Arch Toxicol 85: 1407-1417, 2011.

24. Chipuk JE, McStay GP, Bharti A, et al: Sphingolipid metabolism cooperates with BAK and BAX to promote the mitochondrial pathway of apoptosis. Cell 148: 988-1000, 2012.

25. Gu B, Zhang GF, Li LY, et al: Human herpesvirus 6A induces apoptosis of primary human fetal astrocytes via both caspase-dependent and -independent pathways. Virol J 8: 530, 2011.

26. Zhang T, Li Y, Park KA, et al: Cucurbitacin induces autophagy through mitochondrial ROS production which counteracts to limit caspase-dependent apoptosis. Autophagy 8: 559-576, 2012.

27. Rodríguez-Blanco J, Martín V, García-Santos G, et al: Cooperative action of $\mathrm{JNK}$ and $\mathrm{AKT} / \mathrm{mTOR}$ in 1-methyl-4-phenylpyridinium-induced autophagy of neuronal PC12 cells. J Neurosci Res 90: 1850-1860, 2012.

28. LiS,Zhou Y,Fan J,etal: Heat shock protein 72 enhances autophagy as a protective mechanism in lipopolysaccharide-induced peritonitis in rats. Am J Pathol 179: 2822-2834, 2011.

29. Marquez RT and Xu L: Bcl-2: Beclin 1 complex: multiple, mechanisms regulating autophagy/apoptosis toggle switch. Am J Cancer Res 2: 214-221, 2012.

30. Yeh CH, Hsu SP, Yang CC, et al: Hypoxic preconditioning reinforces HIF-alpha-dependent HSP70 signaling to reduce ischemic renal failure-induced renal tubular apoptosis and autophagy. Life Sci 86: 115-123, 2010. 